\title{
Zinc Selenide-Based Schottky Barrier Detectors for Ultraviolet-A and Ultraviolet-B Detection
}

\author{
V. Naval, ${ }^{1}$ C. Smith, ${ }^{1}$ V. Ryzhikov, ${ }^{2}$ S. Naydenov, ${ }^{2,3}$ F. Alves, ${ }^{1}$ and G. Karunasiri ${ }^{1}$ \\ ${ }^{1}$ Department of Physics, Naval Postgraduate School, Monterey, CA 93943, USA \\ ${ }^{2}$ Department of Radiation Instruments, Institute of Scintillation Materials, 61001 Kharkov, Ukraine \\ ${ }^{3}$ Theoretical Department, Institute of Single Crystals, 61001 Kharkov, Ukraine \\ Correspondence should be addressed to G. Karunasiri, karunasiri@nps.edu
}

Received 1 September 2010; Revised 30 October 2010; Accepted 1 November 2010

Academic Editor: Xian Cao

Copyright (c) 2010 V. Naval et al. This is an open access article distributed under the Creative Commons Attribution License, which permits unrestricted use, distribution, and reproduction in any medium, provided the original work is properly cited.

Wide-bandgap semiconductors such as zinc selenide ( $\mathrm{ZnSe}$ ) have become popular for ultraviolet (UV) photodetectors due to their broad UV spectral response. Schottky barrier detectors made of ZnSe in particular have been shown to have both low dark current and high responsivity. This paper presents the results of electrical and optical characterization of UV sensors based on $\mathrm{ZnSe} / \mathrm{Ni}$ Schottky diodes fabricated using single-crystal ZnSe substrate with integrated UV-A (320-400 nm) and UV-B (280-320 nm) filters. For comparison, characteristics characterization of an unfiltered detector is also included. The measured photoresponse showed good discrimination between the two spectral bands. The measured responsivities of the UV-A and UV-B detectors were $50 \mathrm{~mA} / \mathrm{W}$ and $10 \mathrm{~mA} / \mathrm{W}$, respectively. A detector without a UV filter showed a maximum responsivity of about $110 \mathrm{~mA} / \mathrm{W}$ at $375 \mathrm{~nm}$ wavelength. The speed of the unfiltered detector was found to be about $300 \mathrm{kHz}$ primarily limited by the RC time constant determined largely by the detector area.

\section{Introduction}

Schottky barrier photodetectors using wide-bandgap semiconductors have been fabricated for detection of ultraviolet radiation [1-19]. The materials used include SiC [7], III$\mathrm{V}$ nitrides $[8,9]$, and II-VI compounds such as ZnSe [1019]. Previous studies by Bouhdada et al. showed that the wide-bandgap ZnSe has a promising spectral response on detection of short wavelengths [15]. Though other widebandgap semiconductor materials are readily available, $\mathrm{ZnSe}$ has advantages due to its broader wavelength response in the UV spectral range compared to the nitrides [15]. The responsivity of $\mathrm{ZnSe}$ detectors was measured to be in the 0.1 A/W range while detectivity as high as $1 \times 10^{12} \mathrm{~cm}(\mathrm{~Hz})^{1 / 2} / \mathrm{W}$ was reported $[13,15]$. In addition, most wide-bandgap III-V compounds suffer from the lack of lattice-matched substrates on which to grow them while ZnSe is closely lattice matched to GaAs substrates, and high crystalline quality can thus be obtained [20]. ZnSe Schottky diodes have been shown to have a relatively low leakage current which translates into a higher signal-to-noise ratio [17]. The response of
ZnSe Shottky detectors was also analyzed to determine various decay mechanisms after light excitation [14]. In this paper, fabrication and characterization of ZnSe/Ni Schottky barrier photodetectors operating in the UV-A and UV-B spectral ranges are described. The detectors were fabricated on $1 \mathrm{~mm}$ thick n-type (aluminum doped) single crystal ZnSe substrate.

\section{Device Fabrication}

The structure of the $\mathrm{ZnSe} / \mathrm{Ni}$ Schottky diode sensor is shown schematically in Figure 1(a). The detector consists of $1 \mathrm{~mm}$ thick $\mathrm{ZnSe}$ substrate doped with isovalent donor aluminum with a concentration of about $10^{18} \mathrm{~cm}^{-3}$. The crystal electric resistivity was measured to be about $10^{6} \Omega \cdot \mathrm{cm}$ indicating the donor concentration in the $10^{17}$ to $10^{18} \mathrm{~cm}^{-3}$ range. The ZnSe crystals of $1 \mathrm{~mm}$ thickness and $4 \times 4 \mathrm{~mm}^{2}$ area were first etched using a $\mathrm{CrO}_{3}: \mathrm{HCl}$ mixture (in ratio $2: 3$ ) for $20 \mathrm{~s}$ to remove the oxide layer and accumulated impurities on the surface. They were then washed in deionized water, dried, and loaded into a vacuum chamber for deposition of 


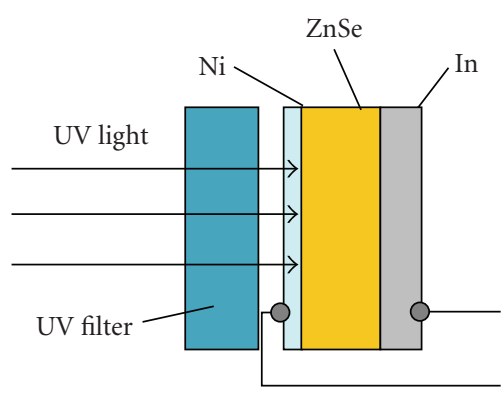

(a)

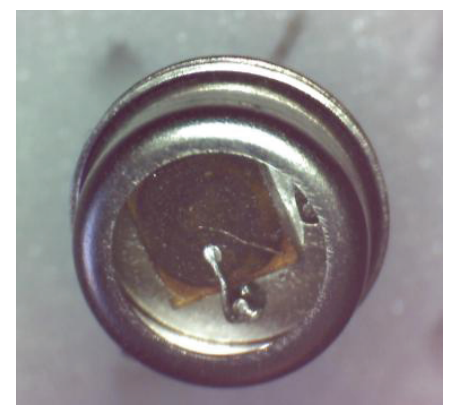

(b)

Figure 1: (a) Schematic diagram of the Schottky diode and (b) packaged detector without UV filter.

metal. A Ni layer of about $10 \mathrm{~nm}$ in thickness was deposited on one side of the crystal through a $3 \times 3 \mathrm{~mm}^{2}$ mask for formation of Schottky barrier while an indium layer of about $100 \mathrm{~nm}$ in thickness was evaporated to the other side to form an ohmic contact. The $10 \mathrm{~nm}$ thick nickel layer is sufficient for formation of a solid metal layer while remaining transparent to UV radiation in the $200-400 \mathrm{~nm}$ range. The Fermi energy of indium is about $3.97 \mathrm{eV}$ [21] which is very close to the electron affinity of zinc selenide $(4.09 \mathrm{eV})$ [22], making indium a relatively good ohmic contact material for ZnSe-based structures. After deposition, the structure formed was put into a furnace, and current output contacts were burnt in. The structure was placed in an appropriate housing that ensured good thermal and electric contact for $\mathrm{Ni}$ and In electrodes. Depending upon the sensor type, the required optical filters were integrated, and the photodetectors were hermetically sealed. Figure 1(b) shows a packaged UV detector without spectral selecting filter attached to it.

For discriminating UV-A and UV-B spectral ranges, glass light filters were used. The choice of light filters is guided by two requirements. The first is cutting the specified optical range from the total UV spectrum. The second is optimum spectral matching of the filter to the spectral sensitivity of the UV sensor to obtain maximum photosensitivity of the detector. For this purpose, we chose a $5 \mathrm{~mm}$ thick UFS-8 glass as UV-A filter, and its transmission characteristics are shown in Figure 2 curve A. For UV-B filters, the required shape of the spectral distribution was obtained by using two glass materials in combination: (1) UFS-2 glass of $2.5 \mathrm{~mm}$ thickness (curve B1 in Figure 2) and (2) ZhS-3 glass

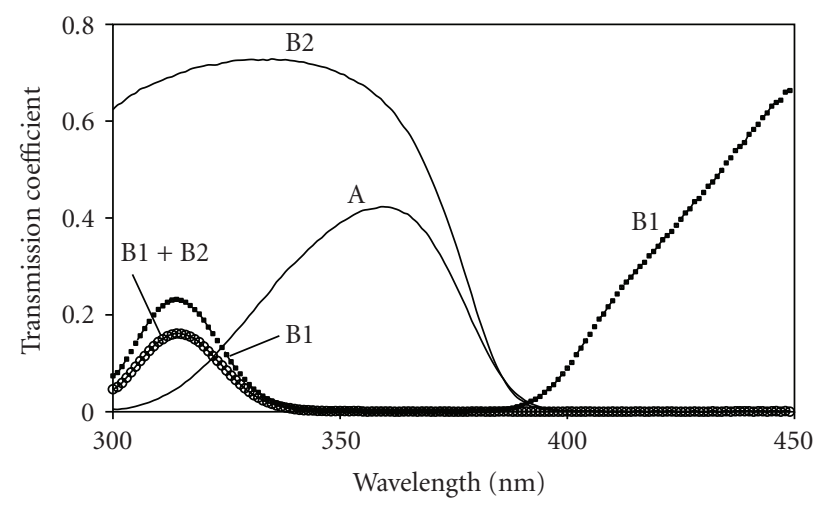

FIgURE 2: Transmission characteristics of optical filters used for discriminating UV-A and UV-B ranges. Filter characteristics for UV-A are shown by curve A (UFS- 8 , thickness $5 \mathrm{~mm}$ ). For UV-B, a combination of two materials was used: UFS-2 with thickness of $2.5 \mathrm{~mm}$ (curve B1), ZhS-3 with thickness of $2.5 \mathrm{~mm}$ (curve B2). The curve $\mathrm{B} 1+\mathrm{B} 2$ shows the transmission of the combined filter.

of $2.5 \mathrm{~mm}$ thickness (curve B1 in Figure 2). The spectral characteristic of the combined filter is shown by the curve $\mathrm{B} 1+\mathrm{B} 2$ in Figure 2. Three different ZnSe:Ni Schottky barrier diodes, one with UV-A, the second UV-B filters, and the third unfiltered, were used in the electrical and optical characterization of this study.

\section{I-V Characteristics}

The current-voltage characteristics of the ZnSe Schottky photodiodes were measured using an Agilent 4145B Semiconductor Parameter Analyzer. The dark or leakage current was measured under dark conditions, wherein the lid of the Agilent 16442A test fixture connected to the parameter analyzer was closed and the photodiode was not exposed to any external light. In this condition, the photodiode has high resistance. The measured I-V characteristics of one of the detectors with and without illumination are shown in Figure 3 which clearly shows the expected diode-like behavior. A relatively low-leakage current of about $20 \mathrm{nA}$ was observed at a reverse bias of $0.5 \mathrm{~V}$. It can be clearly seen in Figure 3 that at around $+0.4 \mathrm{~V}$, the leakage current rapidly increases due to breakdown of the diode. In addition to the dark current, I-V characteristics were measured under illumination using a flash light as indicated by the dotted line of Figure 3 which clearly shows the contribution from generation of photocurrent. The light was illuminated through the $10 \mathrm{~nm}$ thick $\mathrm{Ni}$ layer, and the generation of photocurrent is primarily due to generation of photoexcited electron-hole pairs in the semiconductor.

\section{Photoresponse}

The spectral responses of the three diode types were measured using a computer controlled monochromator fitted with a UV-enhanced grating and a light source. A standard lock-in technique with a mechanical chopper was used for recording photocurrent as a function of wavelength. 


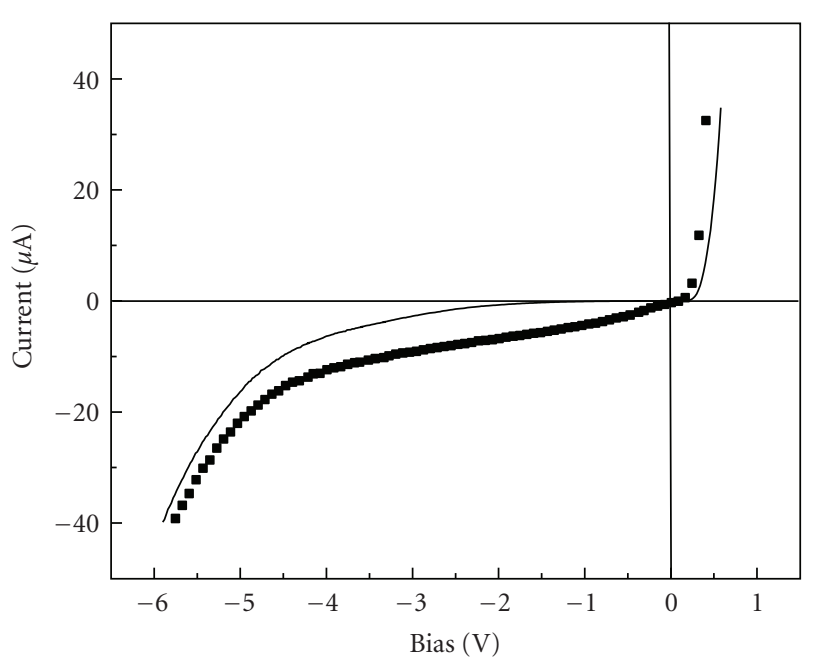

- Dark current

- With illumination

FIGURE 3: Current-voltage characteristics of unfiltered photodiodes in both dark and with illumination using a flash light. Active area is about $3 \times 3 \mathrm{~mm}^{2}$.

The photocurrents generated by the Schottky detector and a calibrated UV-enhanced Si p-i-n diode with no external bias were simultaneously measured through the use of a beam splitter. The responsivities $(\mathrm{A} / \mathrm{W})$ of the three sensors were estimated using the measured photocurrent and power determined from the calibrated Si diode. Figure 4 shows the measured responsivity as a function of wavelength for the unfiltered Schottky detector covering the spectral range from $250-475 \mathrm{~nm}$. The shutting off of photoresponse at above approximately $475 \mathrm{~nm}$ corresponds to photon energy of $2.61 \mathrm{eV}$ which agrees well with the published bandgap of $\mathrm{ZnSe}$ of around $2.7 \mathrm{eV}$ [20]. The maximum responsivity of $110 \mathrm{~mA} / \mathrm{W}$ was observed near $375 \mathrm{~nm}$. At high photon energies, the responsivity was found to decrease mostly due to lack of UV light reaching the depletion region of the semiconductor because of a combination of high reflection and absorption in the metal. In addition, surface recombination in $\mathrm{ZnSe}$ near the interface can play a role in reducing the responsivity since strong absorption occurs near the junction at the shortwave end of the UV spectrum [19].

The responsivities of the UV-A and UV-B detectors as a function of wavelength are shown in Figure 5. It can be seen that the filters provided good discrimination between the two UV bands. The peak responsivity of $50 \mathrm{~mA} / \mathrm{W}$ at about $370 \mathrm{~nm}$ was observed for the UV-A detector while a relatively low responsivity of about $10 \mathrm{~mA} / \mathrm{W}$ at $320 \mathrm{~nm}$ was measured for the UV-A detector. The reduction of peak responsivity of the UV-A detector is primarily due to reflection and absorption losses of light of the filter used. It can be seen from the transmission characteristics of the UV-A filter shown in Figure 2 that it transmits about $50 \%$ of the incident light. In the case of the UV-B detector, reduction of responsivity comes from two factors: (a) reduction of responsivity at short

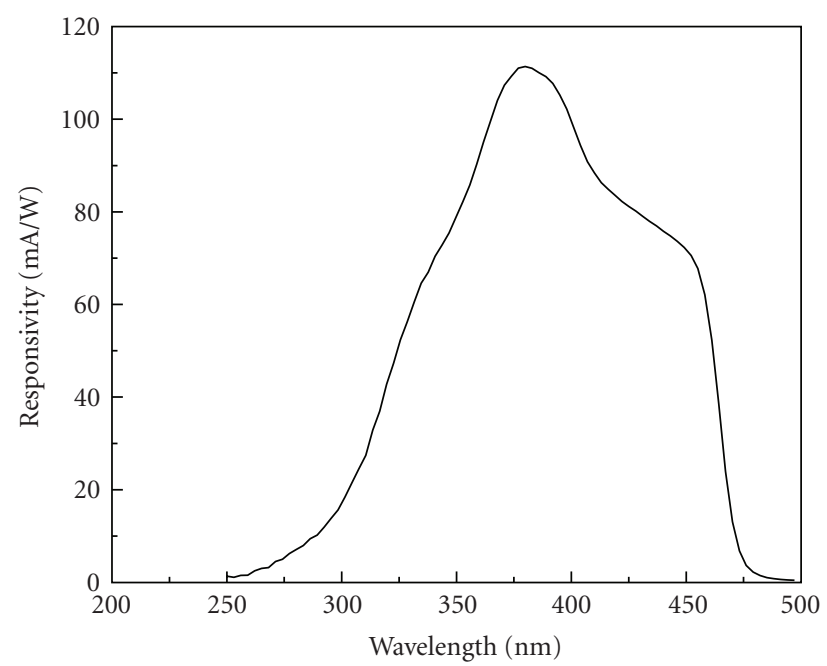

FIGURE 4: Spectral responsivity as a function of wavelength for the unfiltered photodiode.

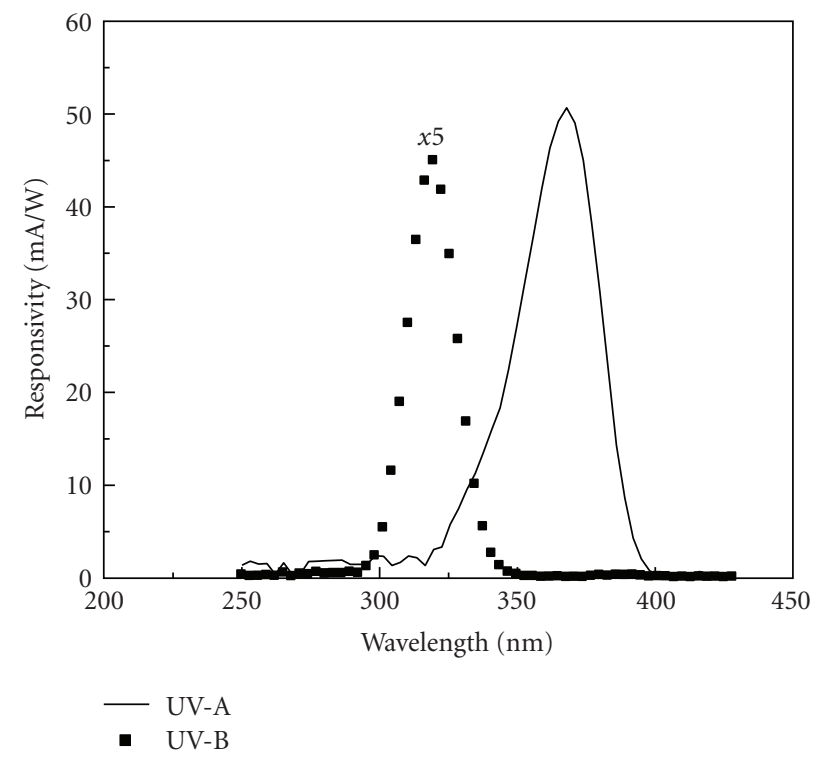

FIGURE 5: Measured spectral responsivity of UV-A and UV-B photodiodes as a function of wavelength.

wavelengths (see Figure 4) and (b) reflection and absorption losses of the UV-A filter transmit about 20\% (see Figure 2).

\section{Speed of Operation}

The speed of operation of the detector was measured in photocurrent mode using a reverse bias of $3 \mathrm{~V}$ and a $100 \Omega$ load resistor. The detector was illuminated using a blue LED connected to a pulse generator with maximum frequency of $30 \mathrm{MHz}$. The unfiltered sensor was used in the measurement due to its high responsivity which increased the photocurrent and hence the voltage across the load resistor. All the detectors have the same physical dimensions except for the external filters attached to UV-A and UV-B detectors. 
The voltage across the load resistor was amplified using a low-noise preamplifier with $1 \mathrm{MHz}$ bandwidth. Figure 6 shows the measured response of the detector as a function of modulation frequency of the LED plotted using log scales for the both axes.

The frequency at the $3-\mathrm{dB}$ cutoff $\left(f_{c}\right)$ was found to be about $280 \mathrm{kHz}$. In order to compare the measured speed of operation of the detector with that determined from the $\mathrm{RC}$ time constant, depletion capacitance of the detector as a function of reverse bias was first determined using $\mathrm{C}-\mathrm{V}$ measurements. The measured capacitance of the detector was found to be about $5 \mathrm{nF}$ at $3 \mathrm{~V}$ reverse bias. The estimated cutoff frequency, $f_{c}=1 /(2 \pi \mathrm{RC})$, with a $100 \Omega$ load resistor was found to be about $318 \mathrm{kHz}$ which is close to that of the measured value of $280 \mathrm{kHz}$. This confirms that the speed is limited by the RC time constant of the detector circuit. Note that the present detectors are not optimized for high speed operation which requires reduction of the device area to reduce the depletion capacitance.

\section{Detectivity}

The detectivity $\left(D^{*}\right)$ of the unfiltered sensor was estimated using the measured dark current and assuming that shot noise is the primary source of noise in the detector. The estimation of the detectivity was done using the formula in (1)

$$
D^{*}=\frac{R \sqrt{A}}{\sqrt{2 e I_{d}}},
$$

where $I_{d}$ is the dark current, $A$ is the area of the photodiode, and $R$ is the responsivity. The area of the diode is $3 \times 3 \mathrm{~mm}^{2}$, and the measured responsivity of $0.11 \mathrm{~A} / \mathrm{W}$ at $375 \mathrm{~nm}$ was used for the estimation. Figure 7 shows the estimated $D^{*}$ as a function of reverse bias voltage. As expected, at high bias a lower $D^{*}$ was obtained due to larger dark current. Note that the detectors were not optimized for achieving low dark current and the values obtained for $D^{*}$ are within the range of the reported values for ZnSe-based Schottky barrier UV detectors $[13,15]$.

\section{Conclusions}

The electrical and optical properties of ZnSe/Ni Schottky barrier photodectors fabricated on single-crystal ZnSe substrates with and without filters for discrimination of UV$A$ and UV-B were measured. The I-V characteristics clearly showed the expected diode-like behavior and a relatively low dark current. As expected, the unfiltered detector showed the largest responsivity since the filters reduce the amount of light entering the sensors. Spectral response of the unfiltered photodiodes showed a broad response in UV spectral range. The detectors with filters clearly showed detection either in UV-A or UV-B ranges with good discrimination. None of the detectors show any response in the visible spectral range due to the large bandgap of $\mathrm{ZnSe}$. The measured values of responsivity for unfiltered photodiodes are in good agreement with previously reported values for similar

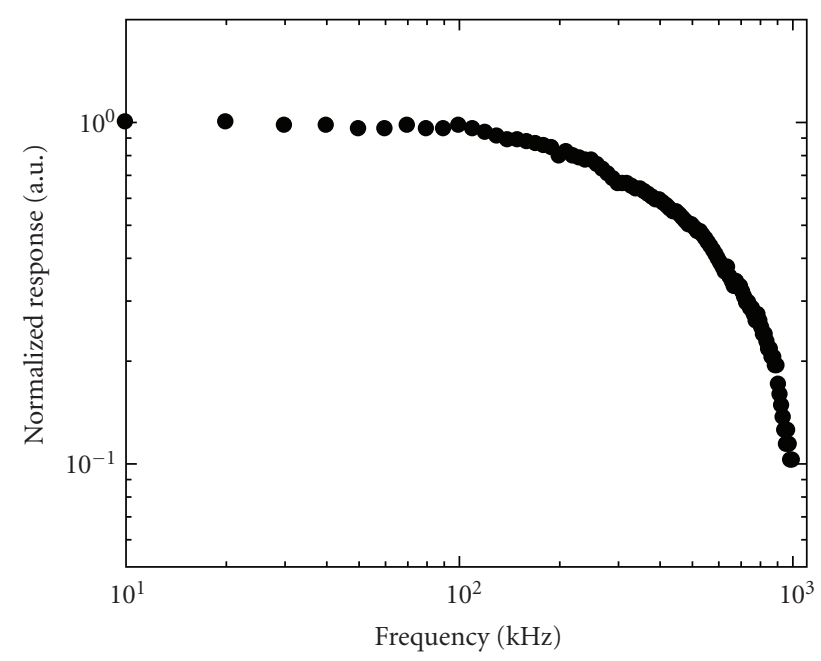

FIGURE 6: Measured photoresponse as a function of frequency using a pulsed blue LED as the excitation source.

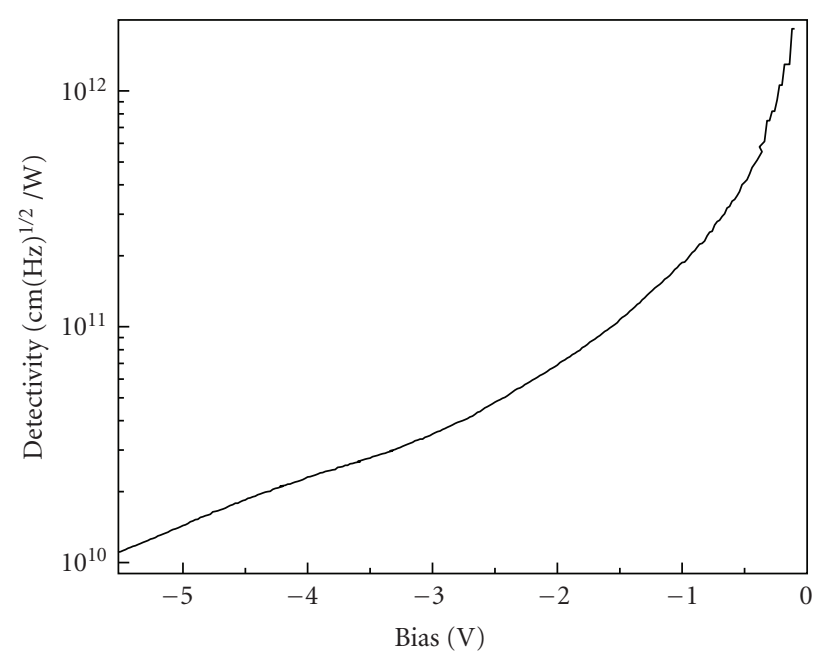

Figure 7: Estimated detectivity $\left(D^{*}\right)$ as a function of bias across unfiltered photodiode.

Schottky diodes. The speed of the detector was found to be about $300 \mathrm{kHz}$ and primarily limited by the RC time constant due to the large area of the detector.

\section{References}

[1] E. Monroy, F. Omnès, and F. Calle, "Wide-bandgap semiconductor ultraviolet photodetectors," Semiconductor Science and Technology, vol. 18, no. 4, pp. R33-R51, 2003.

[2] A. BenMoussa, A. Soltani, U. Schühle et al., "Recent developments of wide-bandgap semiconductor based UV sensors," Diamond and Related Materials, vol. 18, no. 5-8, pp. 860-864, 2009.

[3] S. N. Mohammed, A. A. Salvazdor, and H. Markoc, "Emerging GaN based devices," Proceeding of IEEE, vol. 83, pp. 1306$1355,1995$. 
[4] D. Walker, X. Zhang, A. Saxler, P. Kung, J. Xu, and M. Razeghi, " $\mathrm{Al}_{x} \mathrm{Ga}_{1-x} \mathrm{~N}(0 \leq x \leq 1)$ ultraviolet photodetectors grown on sapphire by metal-organic chemical-vapor deposition," Applied Physics Letters, vol. 70, no. 8, pp. 949-951, 1997.

[5] A. Bouhdada, M. Hanzaz, P. Gibart, F. Omnès, E. Monroy, and E. Muñoz, "Modeling of the spectral response of $\mathrm{Al}_{x} \mathrm{Ga}_{1-x} \mathrm{~N}$ schottky ultraviolet photodetectors," Journal of Applied Physics, vol. 87, no. 12, pp. 8286-8290, 2000.

[6] M. Y. Chen and C. C. Chang, "Integrated a ZnSe MSM photodiode and an InGaP/GaAs hbt on a GaAs substrate for high sensitivity short wavelength photodetector," IEEE Sensors Journal, vol. 9, no. 8, pp. 902-907, 2009.

[7] L. A. Kosyachenko, V. M. Sklyarchuk, and Y. F. Sklyarchuk, "Electrical and photoelectric properties of Au-SiC schottky barrier diodes," Solid-State Electronics, vol. 42, no. 1, pp. 145151, 1998.

[8] L. S. Yu, D. J. Qiao, Q. J. Xing, S. S. Lau, K. S. Boutros, and J. M. Redwing, "Ni and Ti Schottky barriers on n-AlGaN grown on SiC substrates," Applied Physics Letters, vol. 73, no. 2, pp. 238-240, 1998.

[9] E. Monroy, F. Calle, J. L. Pau et al., "Analysis and modeling of $\mathrm{Al}_{x} \mathrm{Ga}_{1-x} \mathrm{~N}$-based Schottky barrier photodiodes," Journal of Applied Physics, vol. 88, no. 4, pp. 2081-2091, 2000.

[10] V. P. Makhnii and V. V. Melnik, "Photoelectric properties of Ni-ZnSe contacts," Semiconductors, vol. 29, pp. 764-766, 1995.

[11] A. Gerhard, J. Nürnberger, K. Schüll et al., "ZnSe-based MBEgrown photodiodes," Journal of Crystal Growth, vol. 184-185, pp. 1319-1323, 1998.

[12] V. P. Makhnii, "Schottky barrier UV photodetectors based on zinc selenide," Technical Physics, vol. 43, no. 9, pp. 1119-1120, 1998.

[13] F. Vigué, P. de Mierry, J. P. Faurie, E. Monroy, F. Calle, and E. Muñoz, "High detectivity ZnSe-based Schottky barrier photodetectors for blue and near-ultraviolet spectral range," Electronics Letters, vol. 36, no. 9, pp. 826-827, 2000.

[14] E. Monroy, F. Vigué, F. Calle, J. I. Izpura, E. Muñoz, and J. P. Faurie, "Time response analysis of ZnSe-based Schottky barrier photodetectors," Applied Physics Letters, vol. 77, no. 17, pp. 2761-2763, 2000.

[15] A. Bouhdada, M. Hanzaz, F. Vigué, and J. P. Faurie, "Electrical and optical proprieties of photodiodes based on ZnSe material," Applied Physics Letters, vol. 83, no. 1, pp. 171-173, 2003.

[16] S. J. Chang, T. K. Lin, Y. K. Su et al., "Homoepitaxial ZnSe MSM photodetectors with various transparent electrodes," Materials Science and Engineering B: Solid-State Materials for Advanced Technology, vol. 127, no. 2-3, pp. 164-168, 2006.

[17] M. Hanzaz, A. Bouhdada, F. Vigue, and J. P. Faurie, "ZnSeand GaN-based schottky barrier photodetectors for blue and ultraviolet detection," Journal of Active and Passive Electronic Devices, vol. 2, pp. 165-169, 2007.

[18] T. K. Lin, S. J. Chang, Y. Z. Chiou et al., "Homoepitaxial ZnSe MIS photodetectors with $\mathrm{SiO}_{2}$ and BST insulator layers," SolidState Electronics, vol. 50, no. 5, pp. 750-753, 2006.

[19] V. P. Makhnii and V. V. Melnik, "Surface barrier diode based on zinc selenide with a passivating zinc oxide film," Technical Physics Letters, vol. 29, no. 9, pp. 712-713, 2003.

[20] V. Bousquet, E. Tournié, and J. P. Faurie, "Defect density in ZnSe pseudomorphic layers grown by molecular beam epitaxy on to various GaAs buffer layers," Journal of Crystal Growth, vol. 192, no. 1-2, pp. 102-108, 1998.

[21] V. S. Fomenko, Handbook of Thermionic Properties, Plenum, New York, NY, USA, 1996.
[22] M. Vos, F. Xu, J. H. Weaver, and H. Cheng, "Influence of metal interlayers on Schottky barrier formation for Au/ZnSe (100) and Al/ZnSe (100)," Applied Physics Letters, vol. 53, no. 16, pp. 1530-1532, 1988. 

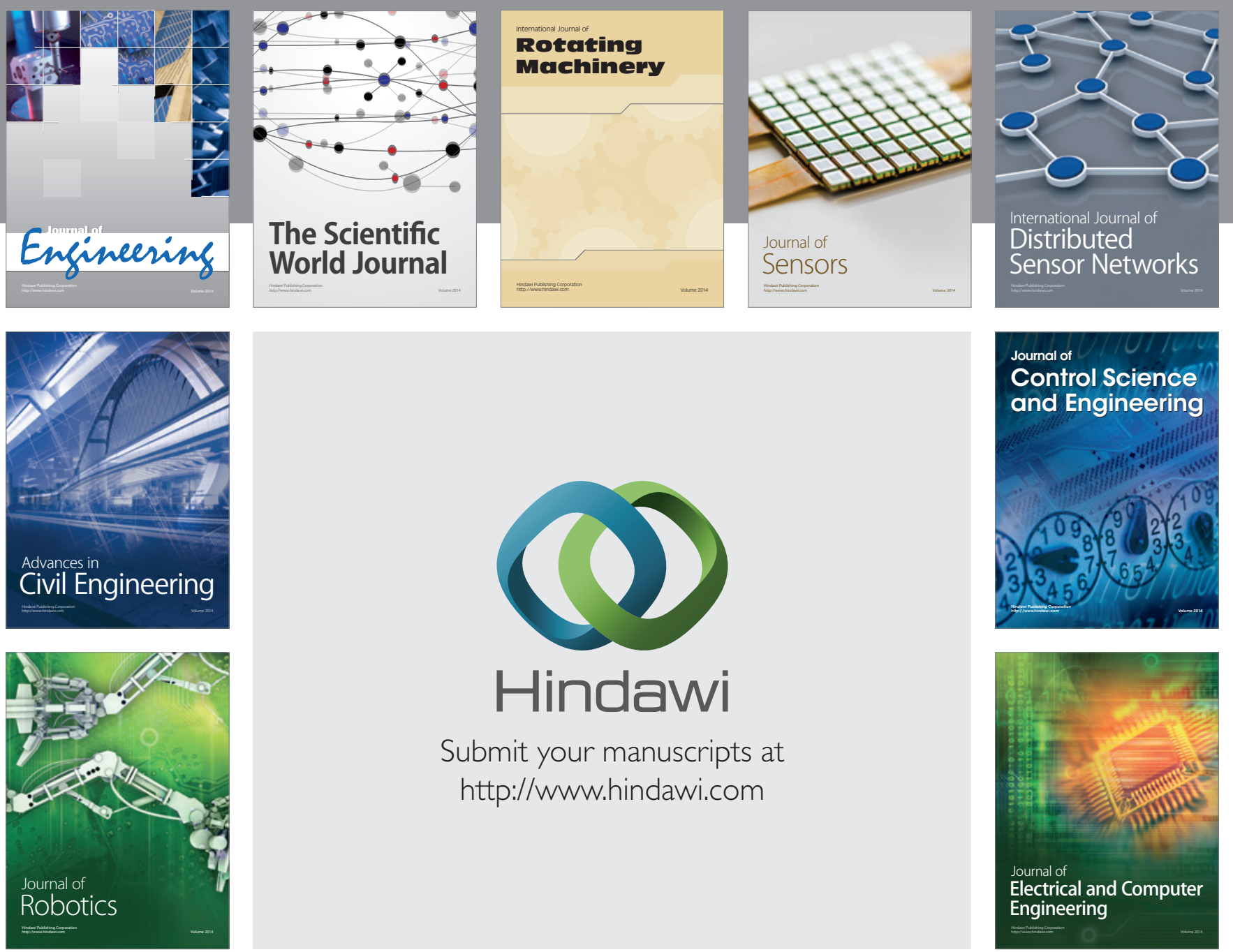

Submit your manuscripts at

http://www.hindawi.com
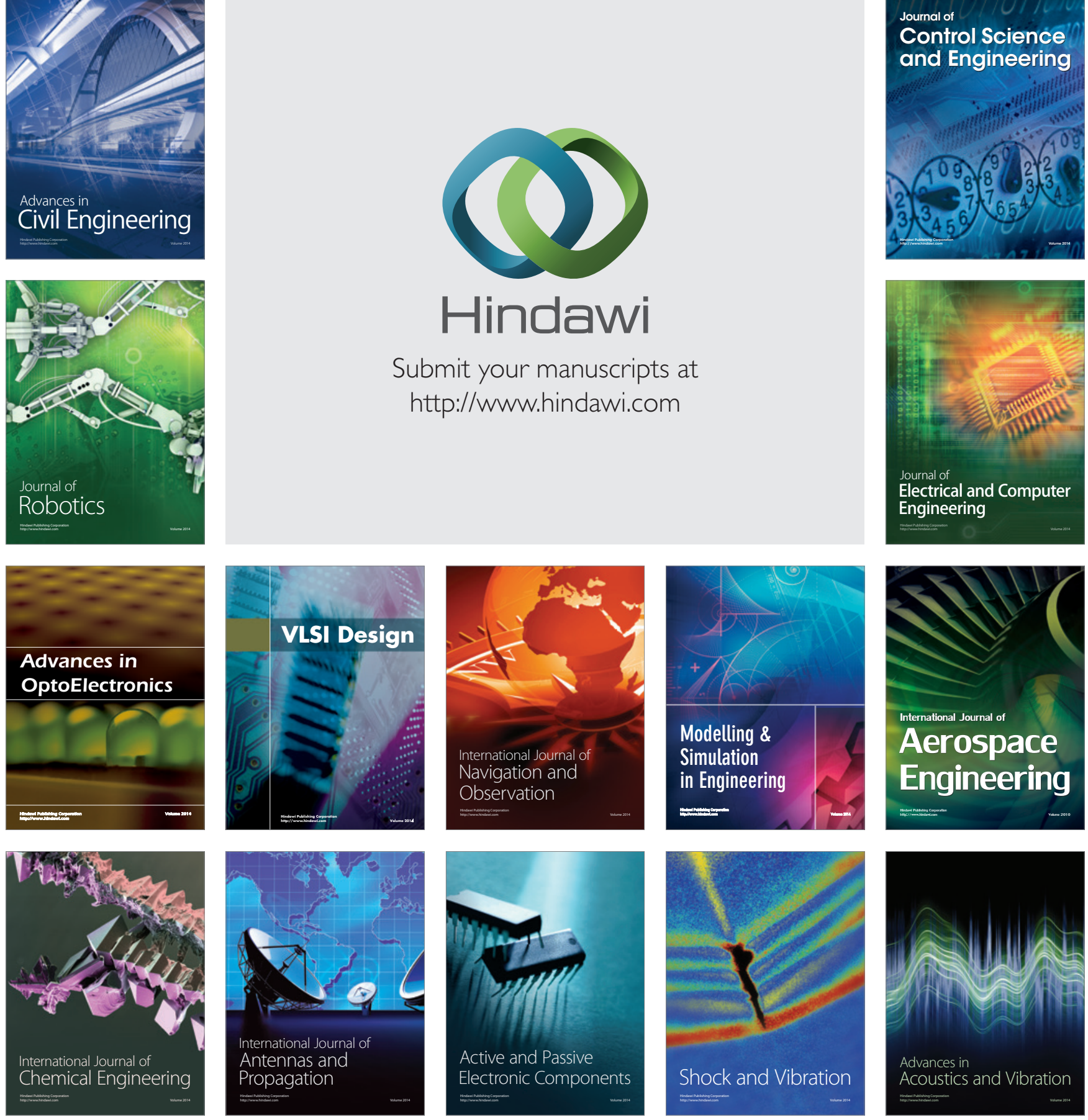